\title{
Editorials
}

\section{Patient and public involvement in general practice research}

Patient and public involvement (PPI) in research has become expected practice by funders and Research Ethics Committees (RECs). ${ }^{1-3}$ Working with public contributors from the outset in designing and undertaking patient-related research aims to ensure that the focus is relevant to, and grounded in, their lived experiences. ${ }^{4}$ Despite sustained UK research policy advocating the importance of $\mathrm{PPI},{ }^{3}$ public contributions are often absent or minimal in reports of primary care research. ${ }^{5}$ In this patient perspectives edition of the $B J G P$, only two papers report on public contributions to the design and execution of the research. ${ }^{6,7}$ When PPI input is described, research reports are rarely specific about the input of public contributors along the way; how their concerns, reviews, suggestions, and objections have actually shaped or changed the course of the research. ${ }^{1,4,8} \mathrm{PPI}$ work often remains a 'black box'.

This editorial makes the case that meaningful PPI makes a positive contribution, but the nature and content of PPI work need to be transparent and appropriate. PPI collaboration in research is time consuming and more problematic for both public contributors and researchers than best-practice guidelines suggest. ${ }^{4,9}$ The reasons why general practice research is not designed and undertaken with public contributors are numerous, including insufficient resources and a desire to avoid complicating a project., ${ }^{2,8}$ We draw on our experience as three researchers and one PPI group member to highlight some of the key challenges and practicalities involved.

\section{DIFFERENT APPROACHES}

The impact of public contributions on research quality remains disputed and the evidence evaluating its impact is limited. $3,5,10$ The literature highlights three broad approaches to the involvement of public contributors in research. The first is an ideological democratic model, in which public contributors are empowered to help decide research priorities, questions, and methods, and carry out data collection, analysis, and dissemination. 4,10 The second is an outcomeoriented model, in which public contributors actively work with researchers to improve the quality and relevance of researcher-led studies. ${ }^{2.5}$ The third is a tokenism model, in which public contributors are only nominally engaged in order to meet external expectations or funding requirements. ${ }^{2,4}$

"Effective collaboration with public contributors relies on researchers being open and transparent about how they will incorporate advice and feedback.

Evidence of the impact of public engagement is ambiguous and has been used to either justify or cast doubt on each of these models. The outcome-oriented model of designing research with public contributions, especially those with lived experiences of the research topic, is regularly cited as improving research methods, participant information summaries, study recruitment, and retention. 1,25,11,12 However, there is conflicting evidence on what impact public contributions have on research outcomes or what the effective components of public contributions are. ${ }^{3,10,11}$ PPI work is heavily promoted in research policy despite the lack of clear evidence about the nature and extent of its contribution. 3.10

It is considered best practice to get help from a range of public contributors to understand different perspectives of the research questions and to navigate the practical issues in undertaking the research. ${ }^{4}$ However, finding suitable public contributors who can give meaningful insights is far from easy, especially in primary care, where the contact details of possible contributors are not readily shared. The more specific the research subject, the harder it is to find public contributors with lived experiences to share. Some clinical researchers have approached their existing patients who they know to have first-hand experience of the subject. This can lead to ethical difficulties, with patients finding it difficult to give negative feedback about clinical care. ${ }^{8}$ Patients and caregivers living with serious and debilitating conditions can find contributing to research absorbs their limited time and energy. Although it is easier to access and work with established PPI groups, their members may not have personal experience of the research subject or the sociodemographic characteristics researchers would ideally like. The benefits of gaining the input of established PPI groups with informed but general views needs to be balanced with the more specific input of harder to locate public contributors with lived experience of a research subject.

A related issue is whether to involve 'experienced' or 'novice' public representatives. Public contributors need time and training to understand the research process." 'Novice' public contributors can struggle to understand the research environment and what researchers would like them to contribute. ${ }^{8}$ Conversely, 'experienced' public representatives from established PPI groups are often easier to find and have a clearer understanding of what they are being asked to contribute to ensure the research is appropriate, robust, and makes sense to potential participants. ${ }^{12}$ We have found that working with both 'novice' and 'experienced' public contributors ensures an optimal broad range of advice in the earlier stages of clarifying research questions and initial design issues. More detailed 'experienced' public contributor advice is particularly helpful in the later stages of refining research methods, designing participant-facing material, and subsequent interpretation of emergent data analysis and dissemination.

Financial compensation for public contributors expenses and time is an important consideration. All too often this is inadequately costed, and public contributors give much of their time and expertise for free. ${ }^{4,8}$ The National Institute for Health Research INVOLVE Imww.invo. org.uk) provides guidance on best practice for payments for time, travel, and out-ofpocket expenses, which research funders require in grant applications. The institutional processes for such payments can become a bureaucratic quagmire that is challenging to navigate, leaving public contributors feeling undervalued and awkward when payments are not made promptly. It can be very helpful to all to find a friendly administrator who knows how to keep public contributors paperwork to a minimum.

\section{EFFECTIVE COLLABORATION}

Effective collaboration with public contributors relies on researchers being open and transparent about how they will incorporate advice and feedback. ${ }^{9}$ If public contributors think a research idea is not important or 
could be improved, it is important to listen; how much influence they really have is largely down to the research team. ${ }^{1.8}$ It can be challenging for researchers to involve public contributors in research processes that they have themselves spent considerable time acquiring the research skills to do. ${ }^{2}$ In our experience, decisions concerning research focus and logistics are best shared, with the researchers taking more of a lead concerning methodological decisions, data collection, and analysis.

Reciprocity is an important part of the public contributor and researcher relationship. Both novice and experienced researchers can mistakenly think that they have a largely transactional relationship with public contributors. ${ }^{2}$ It is important to give public contributors space and time to tell their own stories: their past experiences are often the reason they want to contribute to a research project. The time and resources for facilitating the reciprocal relationship need to be factored into research plans from the outset.

One of the most powerful aspects of active involvement of public contributors in research is the reassurance they can provide to RECs. Committees have a responsibility to ensure that research is appropriate and acceptable to potential participants; they can find this challenging in topics where they lack expertise or personal experience. As an example, one of our public contributor colleagues recently accompanied us to an REC meeting, who were considering our general practice end-of-life care study investigating patient and informal carer views and experiences of care. She was able to highlight her views that the study was highly relevant and important, and that it was vital to give patients and their informal carers the opportunity to share their perspectives. The REC welcomed her contribution and warmly supported the study.

\section{EMOTIONAL EFFORT}

Working with public contributors requires significant emotional and administrative effort for everyone involved. ${ }^{2}$ Public contributors are often sharing poignant personal stories and researchers also have to manage their own feelings and emotional responses. The work with public contributors is often delegated to relatively junior researchers who report feeling unprepared for the emotional challenges involved. ${ }^{2}$ This may indicate an assumption of more senior researchers that PPI work is unimportant or does not require their involvement. It is important that senior researchers are directly involved in work with public contributors to ensure that their contributions are fully included throughout the research process and to support junior researchers. It is all too easy for researchers, especially those working outside of their comfort zone, to view working with public contributors cynically and reduce it to a tokenistic 'tick-box' activity. ${ }^{2,8}$

Public contribution to a research study needs to be timely and proportionate. It is important to be clear from the outset why, how, and when public contributions would be useful. ${ }^{8}$ It is our experience that, when adequately supported and resourced, working with public contributors is illuminating, rewarding, and makes a genuine contribution to general practice research studies. ${ }^{1,2,12}$

\section{Ben Bowers,}

PhD Student and Queen's Nurse, Primary Care Unit, Department of Public Health and Primary Care, University of Cambridge, Cambridge.

\section{Roberta Lovick,}

Public Contributor and Member of the Cambridge Palliative and End of Life Care Patient and Public Involvement Group, Primary Care Unit, Department of Public Health and Primary Care, University of Cambridge, Cambridge.

\section{Kristian Pollock,}

Professor of Medical Sociology, Nottingham Centre for the Advancement of Research into Supportive Palliative and End of Life Care, School of Health Sciences, University of Nottingham, Nottingham.

\section{REFERENCES}

1. Boote JD, Twiddy M, Baird W, et al. Supporting public involvement in research design and grant development: a case study of a public involvement award scheme managed by a National Institute for Health Research (NIHR) Research Design Service (RDS). Health Expect 2015; 18(5): 1481-1493.

2. Boylan $A-M$, Locock L, Thomson $R$, Staniszewska S. About sixty per cent I want to do it': health researchers' attitudes to, and experiences of, patient and public involvement (PPI) - a qualitative interview study. Health Expect 2019; 22(4): 721-730

3. Russell J, Greenhalgh T, Taylor M. Patient and public involvement in NIHR research 2006-2019. policy intentions, progress and themes. 2019. https://oxfordbrc.nihr.ac.uk/wp-content/ uploads/2019/05/NIHR-and-PPI-reportFeb_2019.pdf (accessed 6 Apr 2020).

4. Harrison JD, Auerbach AD, Anderson W, et al Patient stakeholder engagement in research: a narrative review to describe foundational principles and best practice activities. Health Expect 2019; 22(3): 307-316

5. Blackburn S, McLachlan S, Jowett S, et al. The extent, quality and impact of patient and public involvement in primary care research: a mixed methods study. Res Involv Engagem 2018; 4: 16

6. Daines L, Morrow S, Wiener-Ogilvie S, et al. Understanding how patients establish strategies for living with asthma: a qualitative study in UK primary care as part of IMP2ART. Br J

\section{ADDRESS FOR CORRESPONDENCE}

\section{Ben Bowers}

Primary Care Unit, Department of Public Health and Primary Care, University of Cambridge, Cambridge CB2 1TN, UK.

\section{Email: bb527amedschl.cam.ac.uk}

\section{Stephen Barclay,}

Senior Lecturer, Primary Care Unit, Department of Public Health and Primary Care, University of Cambridge, Cambridge.

\section{Funding}

Ben Bowers is funded by the National Institute for Health Research (NIHR) School for Primary Care Research. Stephen Barclay is supported by the NIHR Applied Research Collaboration East of England programme. The views expressed are those of the authors and not necessarily those of the NHS, the NIHR, or the Department of Health and Social Care.

\section{Provenance}

Commissioned; externally peer reviewed.

\section{Competing interests}

The authors have declared no competing interests.
Gen Pract 2020; DOl: https://doi.org/10.3399/ bjgp20X708869

7. Hillman SC, Bryce C, Caleyachetty R, Dale J. Women's experiences of diagnosis and management of polycystic ovary syndrome: a mixed-methods study in general practice. $\mathrm{Br}$ $J$ Gen Pract 2020; DOI: https://doi.org/10.3399/ bjgp20X708881.

8. Buck D, Gamble C, Dudley L, et al. EPIC Patient Advisory Group. From plans to actions in patient and public involvement: qualitative study of documented plans and the accounts of researchers and patients sampled from a cohort of clinical trials. BMJ Open 2014; 4(12): e006400.

9. National Institute for Health Research, INVOLVE. Guidance on co-producing a research project. 2018. https://www.invo.org.uk/wp-content/ uploads/2019/04/Copro_Guidance_Feb19.pdf laccessed 6 Apr 2020).

10. Madden M, Speed E. Beware zombies and unicorns: toward critical patient and public involvement in health research in a neoliberal context. Front Sociol 2017; 2: 1-7.

11. Crocker JC, Ricci-Cabello I, Parker A, et al. Impact of patient and public involvement on enrolment and retention in clinical trials: systematic review and meta-analysis. $B M J$ 2018; 363: k4738.

12. Barclay S, Moran E, Boase S, et al. Primary palliative care research: opportunities and challenges. BMJ Support Palliat Care 2019: 9: 468-472. 